\title{
Adaptive Relaying Strategies for Collaborative Spectrum Sensing
}

\author{
Pascale Fouillot and Christophe J. Le Martret \\ Thales Communications \\ 160 Bvd de Valmy, BP-82, 92704 Colombes Cedex, France \\ phone: +(33) 1461325 44, fax: +(33) 146132555 \\ Email: Pascale.fouillot@fr.thalesgroup.com
}

\author{
Ragnar Thobaben \\ School of Electrical Engineering / ACCESS Linnaeus Center \\ Royal Institute of Technology (KTH) \\ SE-100 44 Stockholm, Sweden \\ Email: ragnar.thobaben@ee.kth.se
}

\begin{abstract}
We analyze and compare the performance of two adaptive relaying schemes based on the amplify-and-forward and the decode-and-forward protocols which are employed to improve the performance of collaborative spectrum sensing. As our results show, the proposed schemes allow us to improve the reliability of the communication part of the wireless sensor network. We expect that this in turn will improve the overall sensing performance and the reliability of the spectrum sensing.
\end{abstract}

\section{INTRODUCTION}

Due to the increase of wireless users and the deployment of new wireless services, radio spectrum has become a scarce resource, and efficient spectrum management has become a challenge. It has been recognized that due to the current spectrum regulation it has become increasingly difficult to provide spectrum for new wireless services. At the same time, however, spectral measurements have shown that licensed spectrum is often underutilized.

This observation has motivated the cognitive radio concept [1]. The idea is that wireless users sense for unused spectrum in the licensed frequency bands (so-called spatial or temporal spectrum holes) which can be used for cognitive radio communications, given that harmful interference to the owner of the spectrum is limited. The feasibility of the cognitive radio concept relies however crucially on the reliability of employed spectrum sensing. Research on spectrum sensing has shown that reliable spectrum sensing can only be obtained by cooperative spectrum sensing for example by using a dedicated sensor network. It allows cognitive radios to utilize diversity to cope with the hidden-node problem resulting from the characteristics of radio channels and to aggregate a sufficient amount of signal energy to make reliable decisions on the status of the primary user.

In this paper we focus on communication aspects of wireless sensor networks (WSNs) which are employed for spectrum sensing for cognitive radio systems [2]. The nodes of such a WSN may serve both as sensors and as relays for communications. We assume that the WSN consists of dedicated sensors which are supported in an ad hoc fashion by sensors which are integrated in the user equipment. Depending on the sensing strategy and the number of frequency bands which are scanned by the network medium to large amount of sensing data has to be conveyed in short time through the network. Cooperative communication techniques are necessary to handle the vast amount of data.

In [3] Zhang has presented the benefits of using cooperative relaying strategies in cognitive radio networks to improve the transmission of spectrum information to the secondary network. This concept which uses in addition to the transmitter one or several relays to re-transmit signals to the destination, improves the performance of wireless networks by exploiting time and spatial diversity. During recent years, several relaying schemes have been studied such as amplify-and-forward and decode-and-forward strategies utilizing one [4], two [5], or multiple [6] relay-assisted transmissions and employing different protocols, codes, and receiver processing [4], [7]. For all the different strategies, the performance depends strongly on the relay position. Therefore, a static relaying strategy will only lead to an optimal performance in few configurations (source-relay-destination positions), and it consequently cannot be optimal for all nodes of an ad hoc network. Adaptive relaying protocols that adjust the relaying strategy according to the node positions are therefore in the focus of this paper.

In the following, we discuss two different adaptive relaying schemes and analyze their performance. The first scheme is based on the amplify-and-forward (AF) protocol, and it combines the benefits of a direct transmission with twohop relaying and cooperative relaying based on the Alamouti code. The second scheme is based on the decode-and-forward (DF) protocol. It constructs a distributed multiple parallel concatenated code based on trellis coded modulation (TCM) encoders. Adaptation is carried out by adjusting the assignment of the component encoders to the transmitter and the relay. Related work can be found for example in [8].

The paper is organized as follows: in Section II, we introduce the underlying assumptions for the WSN under consideration. The adaptive relaying protocols are introduced in Section III, and their performances are compared in Section IV. Section V summarizes the conclusions of this paper.

\section{Wireless SENSOR Networks FOR COllaborative SPECTRUM SENSING}

In the following, we specify in detail the properties and characteristics of the underlying system concept and the employed wireless sensor network (WSN). It is based on the 
system definition of the SENDORA system for collaborative spectrum sensing [2].

\section{A. Concept of the SENDORA System}

The SENDORA system considers three different networks, a primary network, a secondary network, and a WSN. The primary network is a licensed network with underutilized resources. The secondary "cognitive" network is an unlicensed network which is however allowed to use the free resources of the primary network for its communications. The WSN is employed to track unused frequencies in the primary network and report it to the secondary network.

The resource allocation in the secondary network is coordinated by a fusion center based on the sensing information provided by the WSN. Depending on the architecture the fusion center is implemented either as a central system entity or it may be implemented locally in the cluster heads if a distributed architecture is chosen. For the centralized system layout, we assume that the sensors report directly to the fusion center (probably in a multi-hop fashion). The sensors may even be connected directly to the backhaul in this case. For the decentralized case, we assume that the network is split into clusters. The cluster heads take local decisions and exchange aggregated information with other clusters.

It is foreseen that the SENDORA system will be applied to provide nomadic broadband access in urban/suburban areas.

\section{B. Collaborative Spectrum Sensing}

Coordinated by the fusion center, the sensors sense a set of frequency bands. The sensors use either a simple energy detector or more complex feature based detectors. Based on their sensing observations, the sensors generate for each band a decision statistic for the primary user activity (for example loglikelihood ratios, LLR). The LLRs are quantized and organized in packets which are then forwarded through the network to the fusion center. The fusion center combines the sensing information provided by several sensors in order to make a reliable decision on the occupancy of the frequency bands. As the study in [9] shows a low number of quantization bits (e.g., 4 bits) is sufficient to obtain a reliable decision. In general, the gain of collaborative spectrum sensing compared to a singlesensor scenario can be attributed to the diversity. In order to achieve the desired diversity it is sufficient to combine the decision statistics of a relatively low number of sensors.

\section{Sensor and Channel Characteristics for the WSN}

In the following we give a short summary on the working assumptions underlying the SENDORA system.

a) Sensor Characteristics: The nodes in the WSN (source, destination, and relay), which represent sensors, are assumed to have the same characteristics. We assume that they operate at the carrier frequency $f=2.5 \mathrm{GHz}$, use a $B=10 \mathrm{kHz}$ transmission bandwidth and a transmission power of $P=40 \mathrm{~mW}$. Nodes are equipped with a single omnidirectional antenna with an antenna gain $G=0 \mathrm{dBi}$. The thermal noise power at the receiver, $N_{0}$, is given by

$$
N_{0}=(K \cdot T)_{d B}+B_{d B}+F_{d B},
$$

where $K$ is the Boltzmann constant, $T$ the ambient temperature in Kelvin $\left((K \cdot T)_{d B}=-174 \mathrm{dBm} / \mathrm{Hz}\right), B_{d B}$ the bandwidth defined above (in $\mathrm{dB}$ ) and $F_{d B}$ the receiver noise factor (in $\mathrm{dB}$ ). Assuming a receiver noise factor of 7 $\mathrm{dB}$, a receiver thermal noise power level of $-127 \mathrm{dBm}$ (i.e. $1.99 \cdot 10^{-16} \mathrm{~W}$ ) is obtained.

b) Channel Characteristics: In this study, we consider the propagation model described below. It considers the impact of noise, path loss, and fading, and it is specialized for the propagation environment of the target scenario of the SENDORA system (nomadic broadband access for urban and suburban areas).

Frequency-Flat Quasi-Static Rayleigh Fading: For a carrier frequency of $2.5 \mathrm{GHz}$ and urban static sensors, the coherence time is about $0.12 \mathrm{~s}$. This is greater than the frame duration used, and therefore, we assume that the channel remains constant during one frame. Considering a typical maximum delay spread of $2 \mu \mathrm{s}$ in an urban macro cell environment, we obtain furthermore a coherence bandwidth of about $500 \mathrm{kHz}$. As the sensors communicate in a $10 \mathrm{kHz}$ bandwidth the channel coherence bandwidth is much larger than the communication bandwidth. We assume therefore a frequency-flat fading channel for the communication between sensors which is modelled by uncorrelated and quasi-static Rayleigh fading.

Path Loss: The signal power is attenuated by path loss. We use the Winner C2 model (urban macro cell) with path loss (in $\mathrm{dB}$ )

$$
P L_{d B}=A \log _{10}(d)+B+C \log _{10}\left(\frac{f}{5.0}\right),
$$

with $A=35.74, B=42.61$, and $C=23$ for $50 \mathrm{~m} \leq d \leq$ $5000 \mathrm{~m}$, where $f$ is the carrier frequency in $\mathrm{GHz}$ and $d$ the distance between the two nodes in meters (m).

In summary, the transmission of a complex data symbol $X$ is modeled as

$$
Y=P L \cdot h \cdot X+W
$$

where $h$ is a zero-mean, proper complex Gaussian process with unit variance, PL considers the path loss, and $W$ is complex additive white Gaussian noise (AWGN) with variance $\sigma^{2}=$ $N_{0}$, where $N_{0}$ is the receiver noise power.

\section{Adaptive Relaying Protocols}

In this section we describe the two adaptive relaying protocols considered in this paper. The first protocol is based on the amplify-and-forward protocol, and its simplicity makes it an interesting candidate for the decentralized scenario in which cooperative relaying is used to improve the communication between the sensors and the cluster heads. The second scheme is based on the decode-and-forward protocol. Due to its good performance resulting from powerful channel coding, it is well suited for communications between cluster heads or communications over larger ranges. 


\section{A. Adaptive Amplify-and-Forward Relaying}

It has been shown in [10] that an amplify-and-forward cooperative transmission scheme does not always outperform the direct transmission. Its performance depends on the relay position, and thus an adaptive solution that selects the best of the two schemes is desirable. This adaptive protocol based on two schemes is denoted $\mathrm{Ad} 2$. We propose here to extend this idea by considering a third transmission scheme, which we call simple relaying. It realizes conventional two-hop relaying based on the AF protocol and without utilizing the direct transmission. We show that this third scheme allows to enhance the global performance compared to the two-scheme solution. In our adaptive scheme (Ad3), the best scheme is selected based on the frame error rate (FER) criterion.

We now detail the three different transmission schemes which are considered by our adaptive AF protocol: the direct transmission (DT), the simple relaying (SR), and the cooperative relaying $(\mathrm{CR})$.

Direct transmission: The data are sent directly from the source to the destination, without using a relay. In this protocol, we implement a simple receiver processing using a matched filter based on the source/destination channel estimate.

Simple relaying: The data sent by the source are relayed by an intermediate node and forwarded to the destination. Thus, the transmission occurs in two phases: first, the source sends the data, then the relay amplifies and forwards the received signal. The destination only takes into account the data received during the second phase. As for the direct transmission, we implement a simple receiver processing using a matched filter based on the respective channel estimates.

Cooperative relaying: We consider a non-orthogonal $\mathrm{AF}$ cooperative relaying protocol; i.e., the source and relay are allowed to transmit simultaneously toward the destination. In a traditional MIMO system based on the Alamouti space time code (STC), each row of the $2 \times 2$ coding matrix represents data to be sent by each antenna at each time period. Similarly, in a cooperative relaying system employing a distributed Alamouti STC [11], each row of the coding matrix refers to the data to be sent by both the source and the relay during the second phase. The source, thus, transmits the first row of the coding matrix during the first phase. During the second phase, the relay forwards the first row while the source tranmits the second one. Cooperation can be achieved by letting the destination combine the signal from the two phases or use only the second one, as assumed in this paper.

In order to keep a constant spectral efficiency between the 3 transmission schemes (from the source point of view), we have adapted the channel coding in the following way. Since the DT and the SR consumes only 1 phase resource while the CR needs 2 , we use a $1 / 2$ rate convolutional code for DT and $\mathrm{SR}$, and no coding for the CR.

\section{B. Adaptive Decode-and-Forward Relaying}

For the adaptive decode-and-forward (aDF) protocol, the transmission is carried out in two phases: in the first phase the source broadcasts its codeword, and the relay and the destination listen to the transmission. At the end of the first phase, the relay decodes and generates new redundancy which is transmitted to the destination in the second phase. The redundancy provided by the relay is designed to help the destination to decode the codeword which was received during the first transmission. Note that we do not consider cooperative transmissions by the source and the relay; i.e., the source is silent in the second phase. An extension to the cooperative case is however straightforward.

The aDF protocol is based on a distributed Turbo trelliscoded modulation (TCM) scheme. The underlying code (assuming perfect decoding at the relay) is composed of a number of $N$ parallel TCM encoders which are separated by interleavers. As for Turbo codes the interleavers lead to good code properties and allow us to use iterative decoding at the destination. An adaptive distributed code can now be obtained by allocating a subset of $N_{s}$ component encoders to the source node and the set of the remaining $N_{r}=N-N_{s}$ component encoders to the relay. In the following we assume that the rate $R$ of the overall system is fixed. We assume that all component codes have the same code rate $R_{c}$ such that $R=R_{c} / N$ following from parallel code structure. We allow furthermore for two different types of component encoders at the source and the relay and refer to them as type-S encoders and type- $\mathrm{R}$ encoders respectively. The resulting code structure is illustrated in Figure 1.

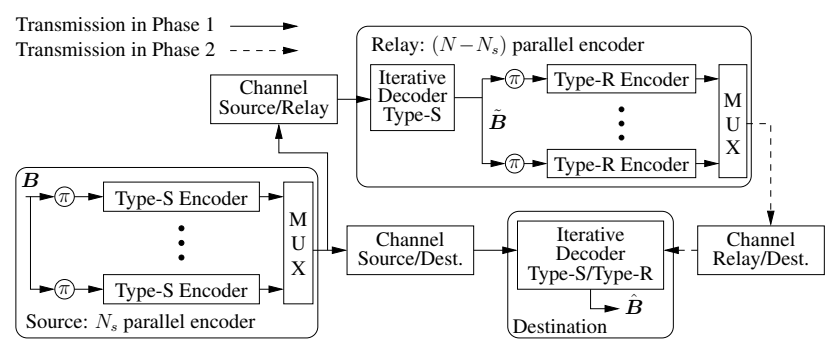

Fig. 1. Code structure of the distributed adaptive decode-and-forward scheme.

Based on this structure, a simple adaptive protocol can be obtained by adapting the number of component encoders allocated to the source $N_{s}$ depending on the position of the relay and the channel conditions. It is equivalent to varying the fraction of channel uses allocated for the first and the second phase which are given by $N_{s} / N$ and $1-N_{s} / N$, respectively. An adaptation in this way is reasonable since for example in a situation where the relay is close to the source node a high code rate is sufficient to make the relay error free while the relay needs at the same time a sufficiently low code rate to protect its transmission to the destination.

The performance of this adaptive coding scheme is evaluated in the following section considering a system with overall rate $R=1 / 2$ and a total number of $N=6$ component encoders. The component encoders used by the source node are given by an 8 -state recursive TCM encoder with rate $R_{c}=3$ using a 16-QAM constellation. The relay employes simple 2-state, non-recursive TCM encoders (again with rate 
(a)

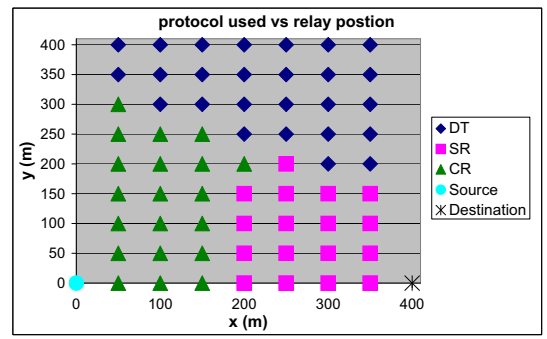

(b)

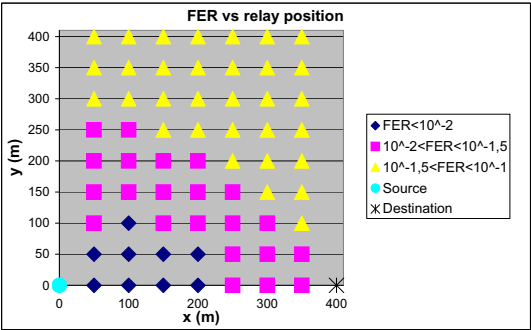

(c)

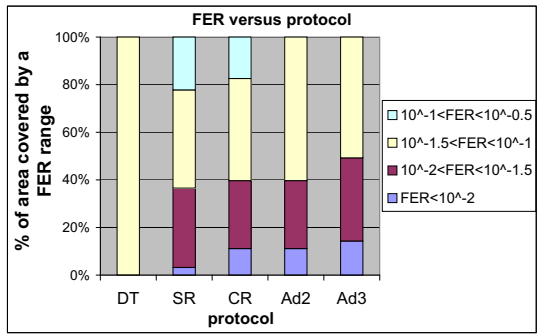

Fig. 2. Performance of the adaptive amplify-and-forward protocol: mode selection for different relay positions in (a), overall frame error rate in (b), and gain in coverage area in $(c)$.

$R_{c}=3$ ) which are matched to the codes employed by the source node using the EXIT charts method.

\section{Simulation Results}

\section{A. Scenario description}

We start our analysis by describing the scenario that is used in our experiments. We are considering sensor and channel characteristics as described in Section II-C and the scenario shown in Figure 3. In the simulations, the distance between the source and the destination is set to $400 \mathrm{~m}$, and the relay moves in a $300 \mathrm{~m}$ by $400 \mathrm{~m}$ area in steps of $50 \mathrm{~m}$. The performance of the adaptive schemes will be evaluated in terms of frame error rate (FER), and the results are obtained from MonteCarlo simulations

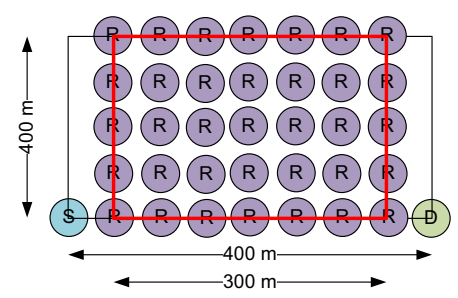

Fig. 3. Scenario for the performance evaluation.

\section{B. Adaptive Amplify-and-Forward Relaying}

The results obtained for the adaptive AF protocol are shown in Figure 2. Figure 2(a) illustrates which transmission strategy is selected by the adaptive protocol for the different relay locations. As we can see cooperative relaying is selected for small source-relay distances, whereas simple relaying is preferable for small relay-destination distances. When the relay is far from both source and destination the direct transmission becomes the best solution.

Figure 2(b) presents the FER obtained at the destination for each relay position with the adaptive amplify-and-forward protocol. The best performance $\left(\mathrm{FER}<10^{-2}\right)$ is obtained when the relay is close to the source (in the first half of the source destination distance) and near to the source-destination axis. The FER then increases when the relay moves away from the source and the destination until it reaches the FER of the direct transmission.
To illustrate the gain of the adaptive AF protocol compared to the static ones, we consider in the following for each protocol the percentage of area in which the relay can be set to obtain a FER in a given range at the destination. The results are shown in Figure 2(c). We can conclude that SR and CR improve performance in $36 \%$ and $40 \%$ of the cases respectively, but they also decrease the performance in $22 \%$ and $17 \%$ of the cases which is a strong drawback. The Ad2 leads to the same performance as the CR and avoids its main drawback limiting the FER to the direct transmission's one. However, the Ad3 takes advantage of both SR and CR and allows us to improve the performance in $50 \%$ of the cases and to limit the FER to the one of the direct transmission in the other $50 \%$ of the cases.

\section{Adaptive Decode-and-Forward Relaying}

In the following we analyze the performance for the adaptive decode-and-forward protocol introduced in Section III-B. Since stronger codes are used in this experiment as compared to the previous experiment, the transmission power has been reduced by $6 \mathrm{~dB}$. The results are depicted in Figure 4 .

Figure 4(a) shows a contour plot for the frame error rate obtained by the adaptive protocol. Note that decode-andforward relaying is only a reasonable solution if the sourcerelay distance $d_{s r}$ is lower than the source-destination distance $d_{s d}$. In this case, the destination will have on average a higher probability of decoding. For this reason Figure 4(a) considers only results for relay positions $(x, y)$ that satisfy $d_{s r}=\sqrt{x^{2}+y^{2}} \leq d_{s d}$.

From Figure 4(a) we can conclude that the best performance $\left(\mathrm{FER} \leq 5 \cdot 10^{-3}\right)$ is obtained if the relay is located in the region $\mathcal{R}^{+}=\{(x, y): 0 \leq x \leq 200 \mathrm{~m},|y| \leq 200 \mathrm{~m}\}$. If the relay is moved further away from the source, the performance deteriorates as expected. However, almost for the entire region of interest $\mathcal{R}_{0}=\left\{(x, y): \sqrt{x^{2}+y^{2}} \leq d_{s d}\right\}$ the frame error rate is below $10^{-1}$.

In order to demonstrate the impact of the code parameter $N_{s}$ (i.e. the number of component encoders employed by the source node), Figure 4(b) shows the value of $N_{s} \in\{1, \ldots, 6\}$ over the position of the relay. Here, the value of $N_{s}$ is encoded by the grey scale where $N_{s}=1$ is represented in black (i.e., the highest code rate possible at the source-node), and $N_{s}=6$ is represented in white (i.e., direct transmission/no relaying). 
(a)

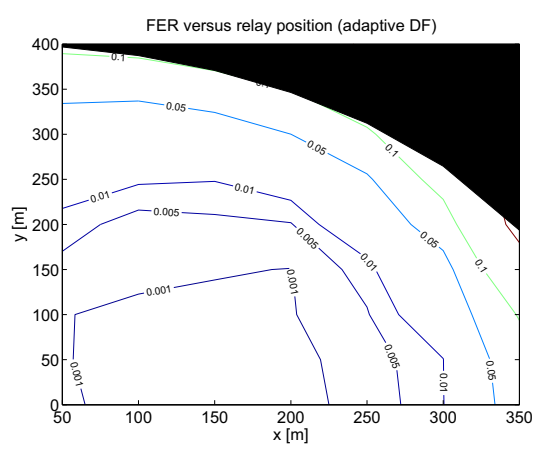

(b)

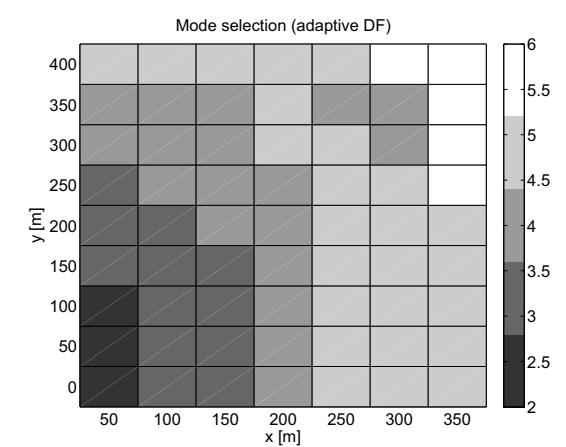

Fig. 4. Performance of the adaptive decode-and-forward protocol: overall frame error rate in (a) and mode selection for different relay positions in (b).

As we can see from Figure 4(b), the mode $N_{s}=1$ is never selected which is a consequence of the code design; for this setup the codes employed by the relay do not match the code from the source very well from which a lower probability of decoding follows. For the remaining modes $N_{S} \geq 2$, we can see that the mode selection works as expected: the further the relay is away from the source node, the more redundancy has to be spent by the source node. As expected for sourcerelay distances which exceed the source-destination distance the direct transmission is selected. We can see however that if the source-relay distance is approximately equal to the sourcedestination distance slight gains due to the diversity provided by the relay can be obtained.

\section{Discussion}

As the results have shown both adaptive relaying protocols can be appropriately adjusted to different relay positions, and in both cases, it could be observed that we benefit most if the relay is located close to the source node. This result can be utilized to optimize the WSN design of the SENDORA system in order to make the communication part of the WSN as reliable as possible. If we compare furthermore the performance of the adaptive protocols to the performance of the static schemes, we can expect a significantly improved sensing performance due the gained reliability of the WSN.

Due to the strong channel codes which were employed by the DF scheme, we were able to reduce the transmit power by $6 \mathrm{~dB}$ relative to the AF scheme. The price is however an improved complexity of the relay and the destination. We can conclude from this that the adaptive AF protocol is an excellent choice for shorter communication ranges and lowcomplexity relaying. This setup is relevant in the ad hoc part of the network when the sensors cooperate to convey their data to the fusion center. The adaptive DF protocol is on the other hand attractive for longer communication ranges and if a higher decoding complexity is admitted. It can be applied, e.g., for exchanging sensing data between the cluster heads.

\section{CONCLUSION}

In this paper, we have analyzed the performance of adaptive relaying for wireless sensor networks (WSNs) used for collaborative spectrum sensing. We have provided an overview on the target scenario and discussed two adaptive protocols, one based on amplify-and-forward relaying and a second based on decode-and-forward relaying. The results have shown that the adaptive designs improve significantly the performance of the communication part of the WSN. From the increased reliability of the WSN we expect as well an improved sensing performance.

\section{ACKNOWLEDGMENT}

The research leading to these results has received funding from the European Community's Seventh Framework Programme (FP7/2007-2013) under grant agreement $n^{\circ}$ 216076. The Authors would like to acknowledge the contributions of their colleagues from SENDORA Consortium (http://www.sendora.eu).

\section{REFERENCES}

[1] J. Mitola, Cognitive radio: An integrated agent architecture for software defined radio, Ph.D. thesis, Royal Institute of Technology (KTH), Stockholm, Sweden, 2000.

[2] V. Fodor, R. Thobaben, and B. Mercier et al., "Sensor networks for cognitive radio: Theory and system desig," in ICT Mobile Summit, Stockholm, Sweden, June 2008.

[3] Q. Zhang, J. Jia, and J. Zhang, "Cooperative relay to improve diversity in cognitive radio networks," IEEE Communications Magazine, , no. 2, Feb. 2009.

[4] A. Nosratinia, T.E. Hunter, and A. Hedayat, "Cooperative communication in wireless networks," IEEE Communications Magazine, vol. 42, no. 10 , pp. $74-80$, Oct. 2004 .

[5] P. Fouillot, I. Icart, and C. Le Martret, "Performance analysis of a two-relay assisted transmission scheme," in Proc. European Wireless Conference (submitted), 2010.

[6] B. Rankov and A. Wittneben, "Spectral efficient protocols for halfduplex fading relay channels," IEEE Journal on Selec. Areas in Comm., vol. 25, no. 2, 2007.

[7] Z. Zhong, S. Zhu, and G. Lv, "Distributed space-time coding based on amplify-and-forward protocol," in Proc. First International Conf. on Communications and Networking in China, 2006.

[8] Z. Si, R. Thobaben, and M. Skoglund, "A practical approach to adaptive coding for the three-node relay channel," in Proc. of IEEE Wireless Communications and Networking Conference (WCNC), 2010.

[9] S. Chaudhari and V. Koivunen, "Effect of quantization and channel errors on collaborative spectrum sensing," in Proc. 43rd Annual Asilomar Conference on Signals, Systems, and Computers, Pacific Grove, CA, USA, Nov. 2009.

[10] J. Kim and W. Lee, "Cooperative relaying strategies for multi-hop wireless sensor networks," in Proc. Communication Systems Software and Middleware and Workshops, 2008.

[11] J. N. Laneman and G. W. Wornell, "Distributed space-time coded protocols for exploiting cooperative diversity in wireless networks," IEEE Trans. on Information Theory, vol. 49, no. 10, Oct. 2003. 